\title{
Thermodynamical quantities for overlap fermions with chemical potential
}

\section{Christof Gattringer ${ }^{a}$ and Ludovit Liptak ${ }^{* * b}$}

${ }^{a}$ Institut für Physik, Universität Graz, Universitätsplatz 5, 8010 Graz, Austria

${ }^{b}$ Institute of Physics, Slovak Academy of Sciences, Dúbravská cesta 9, 84511 Bratislava 45, Slovak Republic

E-mail: christof.gattringer@uni-graz.at

ludovit.liptak@savba.sk

Recently a formulation of overlap fermions at finite density based on an analytic continuation of the sign function was proposed. We study this proposal by analyzing the energy and number densities for free fermions as a function of the chemical potential and the temperature. Our results show that overlap fermions with chemical potential give rise to the correct continuum behavior.

The XXV International Symposium on Lattice Field Theory

July 30 - August 4, 2007

Regensburg, Germany

\footnotetext{
* Speaker.

† Our research is supported in part by the Slovak Science and Technology Assistance Agency, Grant No. APVT51-005704, and the Grant Agency for Science, Project VEGA No. 2/6068/2006 (L.L.). Participation of the speaker at the conference was enabled by support of the ESF project CEPOS at FMFI of the Comenius Univ. in Bratislava.
} 


\section{Introduction}

In recent years finite temperature and finite density physics has seen a lot of attention from the lattice QCD community. Although so far mainly traditional fermion formulations such as staggered or Wilson fermions are used, it is an important issue to get also exactly chiral fermions, in particular the overlap operator [1], ready for QCD thermodynamics.

In [2] Bloch and Wettig have suggested a formulation of overlap fermions which includes a chemical potential. The proposal is based on introducing the chemical potential as the 4-th component of the gauge field [3] and an analytic continuation of the sign function into the complex plane, which requires new techniques for the evaluation of the overlap operator [4].

In a recent paper [5] we have studied thermodynamical properties of the new proposal by analyzing the energy density for the free case as a function of the chemical potential and the temperature. In this contribution we augment this analysis by computing also the number density and comparing it to the known result for a free fermion gas. Our study indicates that the $\mu$-and $T$-dependence for both quantities, energy and number density, approach the continuum behavior correctly.

\section{Setup of the calculation}

The overlap Dirac operator $D(\mu)$ for fermions with a chemical potential $\mu$ is given as [2]

$$
\begin{aligned}
& D(\mu)=\frac{1}{a}\left[1-\gamma_{5} \operatorname{sign} H(\mu)\right], \\
& H(\mu)=\gamma_{5}\left[1-a D_{W}(\mu)\right],
\end{aligned}
$$

where the sign function may be defined with the spectral theorem. $D_{W}(\mu)$ denotes the Wilson Dirac operator with chemical potential,

$$
\begin{aligned}
D_{W}(\mu)_{x, y} & =\mathbb{1}\left[\frac{3}{a}+\frac{1}{a_{4}}\right] \delta_{x, y} \\
& -\sum_{j=1}^{3}\left[\frac{\mathbb{1}-\gamma_{j}}{2 a} U_{j}(x) \delta_{x+\hat{j}, y}+\frac{\mathbb{1}+\gamma_{j}}{2 a} U_{j}(x-\hat{j})^{\dagger} \delta_{x-\hat{j}, y}\right] \\
& -\frac{\mathbb{1}-\gamma_{4}}{2 a_{4}} U_{4}(x) e^{\mu a_{4}} \delta_{x+\hat{4}, y}-\frac{\mathbb{1}+\gamma_{4}}{2 a_{4}} U_{4}(x-\hat{4})^{\dagger} e^{-\mu a_{4}} \delta_{x-\hat{4}, y} .
\end{aligned}
$$

For later use we distinguish between the lattice spacing $a$ for the spatial directions $(=1,2,3$ directions) and the temporal lattice constant $a_{4}$. We use periodic boundary conditions in the spatial directions and anti-periodic boundary conditions for time. The chemical potential $\mu$ is introduced in the standard exponential form [3].

For $\mu=0$ the Wilson Dirac operator is $\gamma_{5}$-hermitian, i.e., $\gamma_{5} D_{W}(0) \gamma_{5}=D_{W}(0)^{\dagger}$, implying that $H(0)$ is a hermitian matrix. When the chemical potential $\mu$ is turned on, $\gamma_{5}$-hermiticity no longer holds, and $H(\mu)$ is a non-hermitian, general matrix. This fact implies: First, the eigenvalues of $H(\mu)$ are no longer real and the sign function for a complex number has to be defined in the spectral representation of $\operatorname{sign} H(\mu)$. Second, the spectral representation has to be formulated using left- 
and right-eigenvectors. For the sign function of a complex number we use the analytic continuation proposed in [2] and define the sign function through the sign of the real part

$$
\operatorname{sign}(x+i y)=\operatorname{sign}(x) .
$$

For analyzing thermodynamic properties we study the following observables:

1. The energy density

$$
\varepsilon(\mu)=\frac{1}{V}\langle\mathscr{H}\rangle=-\frac{1}{V} \frac{\partial}{\partial \beta} \ln \operatorname{Tr}\left[e^{-\beta(\mathscr{H}-\mu \mathscr{N})}\right]_{\beta \mu=c}=-\left.\frac{1}{V} \frac{\partial \ln Z}{\partial \beta}\right|_{\beta \mu=c} .
$$

2. The number density

$$
n_{p}(\mu)=\frac{1}{V}\langle\mathscr{N}\rangle=\frac{T}{V} \frac{\partial}{\partial \mu} \ln \operatorname{Tr}\left[e^{-\beta(\mathscr{H}-\mu \mathscr{N})}\right]=\frac{T}{V} \frac{\partial \ln Z}{\partial \mu} .
$$

Here $\mathscr{H}$ is the Hamiltonian of the system, $\mathscr{N}$ denotes the number operator, and $\beta=1 / T$ is the inverse temperature (we set the Boltzmann constant equal to 1 ). The derivatives in the definition of $\varepsilon(\mu)$ are taken such that $\beta \mu=c=$ const.

The continuum result for the subtracted thermodynamical quantities of free massless fermions can be calculated using the definition (2.4) and (2.5). The final expressions together with the logarithm of the partition function can be found in textbooks (see, e.g., [6]).

In the path integral formalism the inverse temperature $\beta$ is given by the extent in 4-direction, which on the lattice has the form $\beta=N_{4} a_{4}$. Thus the derivative $\partial / \partial \beta$ in (2.4) is expressed as $N_{4}^{-1} \partial / \partial a_{4}$. The partition function $Z$ is given by the fermion determinant $\operatorname{det} D$ which we write as the product over all eigenvalues $\lambda_{n}$ of $D(\mu)$. After some algebra one obtains

$$
\begin{aligned}
\varepsilon(\mu) & =-\left.\frac{1}{V N_{4}} \frac{\partial \ln \operatorname{det} D}{\partial a_{4}}\right|_{a_{4} \mu=c}=-\left.\frac{1}{V N_{4}} \sum_{n} \frac{1}{\lambda_{n}} \frac{\partial \lambda_{n}}{\partial a_{4}}\right|_{a_{4} \mu=c}, \\
n_{p}(\mu) & =\frac{1}{V N_{4} a_{4}} \frac{\partial \ln \operatorname{det} D}{\partial \mu}=\frac{1}{V N_{4} a_{4}} \sum_{n} \frac{1}{\lambda_{n}} \frac{\partial \lambda_{n}}{\partial \mu} .
\end{aligned}
$$

\section{Evaluation of the eigenvalues}

For the evaluation of (2.6) and (2.7) we need the eigenvalues $\lambda$ of $D(\mu)$ in closed form. This is done in three steps: First, using Fourier transformation, we bring the Dirac operator for free fermions to $4 \times 4$ block-diagonal form,

$$
\widehat{H}=\gamma_{5} h_{5}+i \gamma_{5} \sum_{v} \gamma_{v} h_{v}
$$

with

$$
\begin{aligned}
& h_{5}=1-\sum_{j=1}^{3}\left[1-\cos \left(a p_{j}\right)\right]-\frac{a}{a_{4}}\left[1-\cos \left(a_{4}\left(p_{4}-i \mu\right)\right)\right], \\
& h_{j}=-\sin \left(a p_{j}\right) \quad \text { for } \quad j=1,2,3, \\
& h_{4}=-\frac{a}{a_{4}} \sin \left(a_{4}\left(p_{4}-i \mu\right)\right) .
\end{aligned}
$$


The spatial momenta are given by $p_{j}=2 \pi k_{j} / a N$, where $N$ is the number of lattice points in the spatial directions and $k_{j}=0,1 \ldots N-1$. The momenta in time-direction are $p_{4}=\pi\left(2 k_{4}+1\right) / a_{4} N_{4}$, $k_{4}=0,1 \ldots N_{4}-1$.

In the second step the spectral representation for the sign function of $\widehat{H}$ is constructed. The left- and right-eigenvectors of $\widehat{H}, l_{j}$ and $r_{j}$ are given by

$$
\begin{aligned}
& l_{1}=l_{1}^{(0)}[\widehat{H}+s \mathbb{1}], l_{2}=l_{2}^{(0)}[\widehat{H}+s \mathbb{1}], l_{3}=l_{3}^{(0)}[\widehat{H}-s \mathbb{1}], l_{4}=l_{4}^{(0)}[\widehat{H}-s \mathbb{1}], \\
& r_{1}=[\widehat{H}+s \mathbb{1}] r_{1}^{(0)}, r_{2}=[\widehat{H}+s \mathbb{1}] r_{2}^{(0)}, r_{3}=[\widehat{H}-s \mathbb{1}] r_{3}^{(0)}, r_{4}=[\widehat{H}-s \mathbb{1}] r_{4}^{(0)} .
\end{aligned}
$$

The constant spinors $l_{j}^{(0)}, r_{j}^{(0)}$ are ( $T$ denotes transposition)

$$
\begin{aligned}
& l_{1}^{(0)}=r_{1}^{(0) T}=c(1,0,0,0), l_{2}^{(0)}=r_{2}^{(0) T}=c(0,1,0,0), \\
& l_{3}^{(0)}=r_{3}^{(0) T}=c(0,0,1,0), l_{4}^{(0)}=r_{4}^{(0) T}=c(0,0,0,1) .
\end{aligned}
$$

where $c=\left(2 s\left(s+h_{5}\right)\right)^{-1 / 2}$ and $s=\sqrt{h^{2}+h_{5}^{2}}$. The eigenvectors obey $l_{i} r_{j}=\delta_{i j}$. The corresponding eigenvalues $\alpha_{j}$ of $\widehat{H}$ are

$$
\alpha_{1}=\alpha_{2}=+s, \alpha_{3}=\alpha_{4}=-s .
$$

Using these eigenvalues and eigenvectors we can construct sign $\widehat{H}$ using the spectral

$$
\operatorname{sign} \widehat{H}=\sum_{j=1}^{4} \operatorname{sign}\left(\lambda_{j}\right) r_{j} l_{j}=\frac{\operatorname{sign}(s)}{s} \widehat{H} .
$$

Inserting this result in (2.1), the eigenvalues of the overlap operator at a given momentum are obtained as

$$
\lambda_{ \pm}=\frac{1}{a}\left[1-\frac{\operatorname{sign}\left(\sqrt{h^{2}+h_{5}^{2}}\right) h_{5} \pm i \sqrt{h^{2}}}{\sqrt{h^{2}+h_{5}^{2}}}\right],
$$

where each of the two eigenvalues is twofold degenerate. In the spectral sums (2.6), (2.7) the label $n$ runs over all momenta and the eigenvalues at fixed momentum as given in (3.7). The necessary derivative with respect to $a_{4}$ and $\mu$ is straightforward to compute in closed form, and the spectral sums (2.6), (2.7) can then be summed numerically. The argument of the sign function cannot become purely imaginary on a finite lattice, and no $\delta$-like terms occur. We remark, that after taking the derivative with respect to $a_{4}$, we set $a=a_{4}=1$, i.e., all the results are presented in lattice units.

\section{Results}

We begin the discussion of our results with lattices which have equal length in all four directions, corresponding to zero temperature in the thermodynamic limit. We focus our attention on the subtracted energy density $\varepsilon(\mu)-\varepsilon(0)$ and the number density $n_{p}(\mu)-n_{p}(0)$. The results for various lattice sizes are shown in Fig. 1. According to [6], we expect the data (symbols in Fig. 1) to approach the continuum form

$$
\varepsilon(\mu)-\varepsilon(0)=\frac{\mu^{4}}{4 \pi^{2}} \quad, \quad n_{p}(\mu)-n_{p}(0)=\frac{\mu^{3}}{3 \pi^{2}}
$$



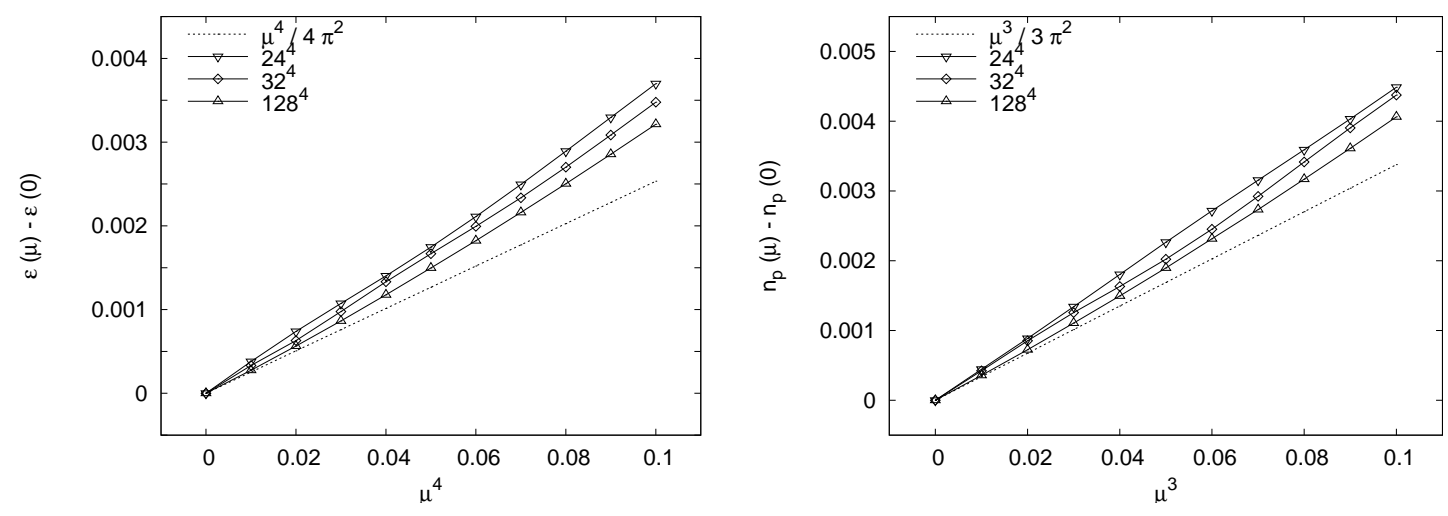

Figure 1: The energy density $\varepsilon(\mu)-\varepsilon(0)$ and the number density $n_{p}(\mu)-n_{p}(0)$ as a function of $\mu^{4}$ and $\mu^{3}$, respectively. The symbols (connected to guide the eye) are for various lattice sizes, the dashed line is the continuum result. All quantities are given in lattice units.

represented by dashed lines in Fig. 1.

We see clearly that both quantities show almost linear dependence on $\mu^{4}$ and $\mu^{3}$, respectively. The figure also demonstrates that with enlarging the lattice the finite size effects become smaller and the lattice results approach the continuum limit. The deviation from the linear dependence is due to the finite extension of the lattice. Furthermore, we can expect that we can see finite temperature effects too.

To study the effects of temperature, we compare our data with the continuum results [6] for the subtracted energy and the number density, now including the leading temperature corrections,

$$
\varepsilon(\mu, T)-\varepsilon(0, T)=\frac{\mu^{4}}{4 \pi^{2}}+\frac{T^{2} \mu^{2}}{2} \quad, \quad n_{p}(\mu, T)-n_{p}(0, T)=\frac{\mu^{3}}{3 \pi^{2}}+\frac{\mu T^{2}}{3} .
$$

Fig. 2 shows the lattice results for finite temperature, using data from $128^{3} \times N_{4}$ lattices with temperature $T=N_{4}^{-1}$ (in lattice units). The approximate linearity of the zero-temperature case receives corrections as the temperature is increased. The temperature-dependent terms have a squareand cubic-root behavior when we plot them as a function of $\mu^{4}$ and $\mu^{3}$, respectively.

In order to study the temperature effect quantitatively, we compare the finite temperature results to the continuum form (4.2). We have to take into account also finite size effects which we build in assuming contributions from higher terms of the expansion in $\mu$. In the case of the energy density, we use the ansatz

$$
\varepsilon(\mu, T)-\varepsilon(0, T)=c_{2} \mu^{2}+c_{4} \mu^{4}+c_{6} \mu^{6}+c_{8} \mu^{8}+\mathscr{O}\left(\mu^{10}\right) .
$$

For the number operator we use an equivalent form with odd powers of $\mu$. The results of the fit for the data used in Fig. 2 and for the largest lattice of Fig. 1 are given in Table 1.

As the table shows, the coefficients approach the continuum values when the temporal extension is increased. In the case of the energy density, the discrepancy for $N_{4}=24$ is approximately $2 \%$ and $9 \%$ and for $N_{128}$ only $0.5 \%$ and $7 \%$. For $N_{4}$ smaller than 24 , the discrepancy for both coefficients is larger, which is due to finite size effects. Nevertheless both, the $\mu^{4}$ - and $\mu^{2} T^{2}$-terms, 

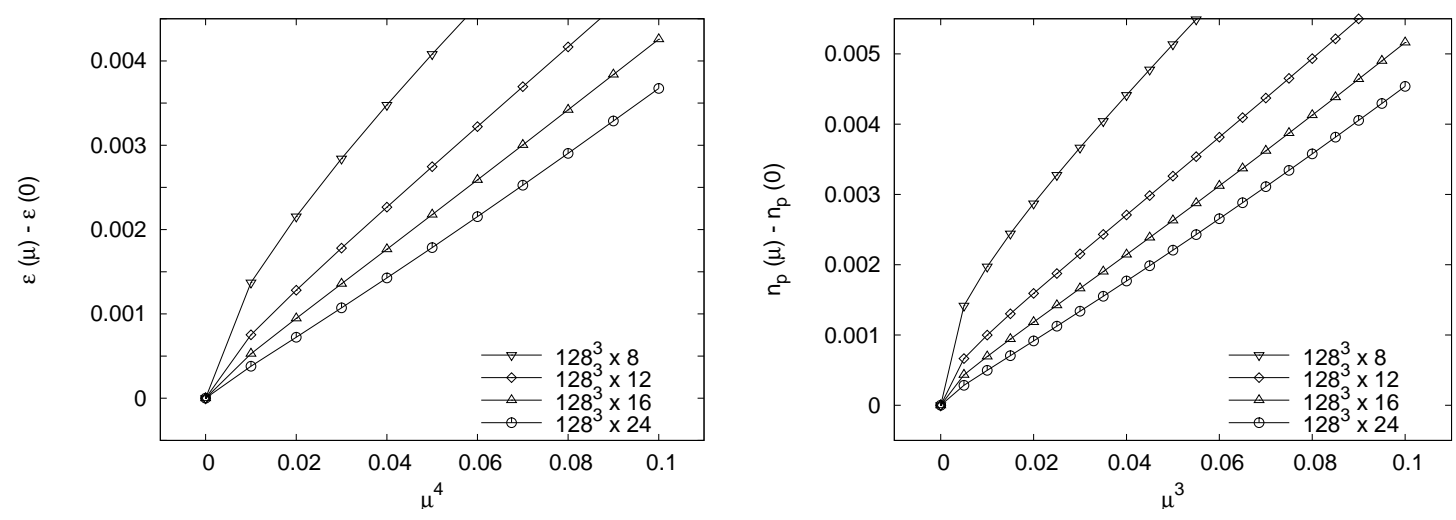

Figure 2: The energy density $\varepsilon(\mu, T)-\varepsilon(0, T)$ and the number density $n_{p}(\mu, T)-n_{p}(0, T)$ as a function of $\mu^{4}$ and $\mu^{3}$, respectively, for finite temperature lattices. All quantities are in lattice units.

are well reproduced. The coefficients $c_{6}$ and $c_{8}$ have magnitudes of $\mathscr{O}\left(10^{-2}\right)$, so their contribution according to (4.3) is irrelevant for $0.1<\mu$ (in lattice units). For the number density we find qualitatively the same behavior with only slightly different numerical values.

Finally, we analyze the $\mu$-dependence for large values of the chemical potential, approaching the cutoff. In this case we compare our data with data obtained for the Wilson fermions and the continuum case. In Fig. 3 we plot the ratio $(\varepsilon(\mu)-\varepsilon(\mu)) / \mu^{4}$. The figure shows that in a certain interval, from zero up to approximately 0.7 in lattice units, both lattice formulations give practically identical results and start to differ only beyond this already relatively large value of $\mu$. The second observation is that up to $\mu \approx 0.6$ the lattice results differ very little from the continuum form and discretization errors grow large only for $\mu \gtrsim 0.6$. Also in a comparison with the data for fermions obtained by blocking [7] one again finds good agreement.

\begin{tabular}{c|ccccc}
\hline \hline$N_{4}$ & $N_{4}^{-2} / 2$ & $c_{2}$ & $c_{4}$ & $D\left(c_{2}\right)$ & $D\left(c_{4}\right)$ \\
\hline 8 & 0.007812 & 0.010125 & 0.03519 & $29 \%$ & $39 \%$ \\
12 & 0.003472 & 0.004125 & 0.03178 & $19 \%$ & $25 \%$ \\
16 & 0.001953 & 0.002192 & 0.02803 & $12 \%$ & $11 \%$ \\
24 & 0.000868 & 0.000947 & 0.02587 & $9 \%$ & $2 \%$ \\
128 & 0.000030 & 0.000032 & 0.02543 & $7 \%$ & $0.5 \%$ \\
\hline \hline
\end{tabular}

Table 1: Results for fits according to (4.3). The spatial volume is always $128^{3}$. The temporal extension $N_{4}$ is given in the first column. In the second column we list the corresponding value of $N_{4}^{-2} / 2$ which is what one expects for the fit coefficient $c_{2}$ in the third column. The coefficient $c_{4}$ is expected to approach the constant value $1 / 4 \pi^{2}=0.02533 . D\left(c_{2}\right)$ and $D\left(c_{4}\right)$ are the relative deviations of $c_{2}$ and $c_{4}$ from the expected continuum values. 


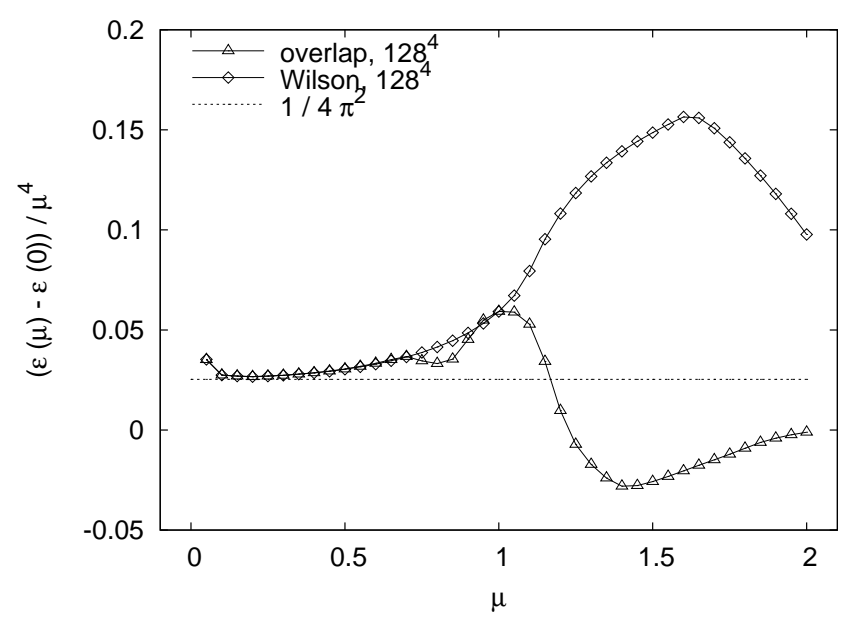

Figure 3: The ratio of $(\varepsilon(\mu)-\varepsilon(\mu)) / \mu^{4}$ as a function of $\mu$. The figure compares overlap fermions with Wilson results and the continuum.

\section{Summary}

We have analyzed thermodynamical quantities for the overlap operator at finite chemical potential obtained by analytic continuation of the sign function into the complex plane as suggested in [2]. The analysis was done for finite and zero temperature lattices. Fits of the data show that the expected continuum behavior is reliably approached and no trace of unphysical $\mu^{2} / a^{2}$ terms was found. We conclude that the suggested introduction of chemical potential [2] provides both, chiral symmetry and the correct description of fermions at finite density.

\section{References}

[1] R. Narayanan and H. Neuberger, Nucl. Phys. B 443 (1995) 305; H. Neuberger, Phys. Lett. B 417 (1998) 141.

[2] J. Bloch and T. Wettig, Phys. Rev. Lett. 97 (2006) 012003 and Pos LAT2006:206 (2006).

[3] P. Hasenfratz and F. Karsch, Phys. Lett. B 125 (1983) 308.

[4] J. Bloch et al, arXiv:0704.3486 [hep-lat].

[5] C. Gattringer and L. Liptak, arXiv:0704.0092 [hep-lat] (to appear in Phys. Rev. D).

[6] J. Kapusta, Finite temperature field theory, Cambridge University Press, Cambridge (1989).

[7] W. Bietenholz and U. J. Wiese, Phys. Lett. B 426 (1998) 114. 